\title{
Endoscopic Closure of a Large Rectovesical Fistula Following Robotic Prostatectomy
}

\author{
Andrew Watts, BS, ${ }^{1}$ Neil J. Kocher, MD, ${ }^{2}$ Eric Pauli, MD, ${ }^{3}$ and Jay D. Raman, MD, FACS ${ }^{4}$
}

\begin{abstract}
Background: Rectovesical fistulae (RVF) are uncommon complications of pelvic surgeries and are a potential cause of significant morbidity. RVF are not typically closed endoscopically but rather require reoperative surgery of the lower pelvis with closure of tract, interposition of fat or omentum, and possible permanent bowel diversion. We present a unique case of a rectovesical fistula developing after robotic prostatectomy that was managed by multimodal multistage endoscopic therapy as an alternative to conventional operative repair.

Case Presentation: A healthy 78-year-old Caucasian man underwent a robot-assisted laparoscopic radical prostatectomy with bilateral pelvic lymph node dissection for high-risk adenocarcinoma of the prostate. The patient's postoperative course was complicated by an unrecognized rectal injury culminating in emergent exploration, abdominal washout, creation of a diverting loop transverse colostomy, and resultant development of a large rectovesical fistula. Given the patient's hostile abdomen and desire for conservative management the fistula was managed through a combined cystoscopic and endoscopic procedure that utilized suturing and clipping to close the fistula. This novel technique was followed by a series of three subsequent endoscopic procedures that enabled us to gradually downsize the fistula over time and ultimately achieve complete closure. The patient's colostomy was eventually reversed with return of bowel continuity.

Conclusion: Although uncommon, RVF are significant complications of pelvic surgery. The presence of abdominal/pelvic adhesions from previous surgeries or patient comorbidities can make open surgical repair extremely challenging or impracticable. Therefore, it is important to recognize and consider the use of endoscopic techniques as potential options for closure of rectovesical fistula in certain situations.
\end{abstract}

Keywords: rectovesical fistula, endoscopic repair, over-the-scope clip system, prostate cancer, prostatectomy, postoperative complication

\section{Introduction and Background}

$\mathbf{R}$ eCTOVESICAL FISTUlae (RVF) are uncommon disease processes with significant patient morbidity and possible mortality rate. ${ }^{1}$ RVF most commonly result from inflammatory conditions, including diverticulitis or Crohn's disease, advanced malignancies, and as complications after pelvic surgery. ${ }^{1,2}$ RVF should be suspected in patients with pneumaturia or fecaluria, especially in the postoperative setting. Surgery is currently the gold standard for managing RVF, but recent advances in endoscopic therapy have provided new options. ${ }^{1}$ In this report, we present a 78 -year-old man who developed a large RVF after a robotic radical prostatectomy for high-risk clinically localized prostate cancer that was managed endoscopically as an outpatient.

\section{Case Presentation}

A 78-year-old Eastern Cooperative Oncology Group 1 Caucasian man with hypertension, history of nephrolithiasis, and high-risk clinically localized prostate cancer was elected for robotic radical prostatectomy with bilateral pelvic lymph node dissection. His intraoperative and initial postoperative course was unremarkable with discharge to home on POD 1. He was readmitted 3 days later with emesis, abdominal distention, and no flatus since surgery suggestive of postoperative ileus. Chest and abdomen X-ray and abdomen CT scan noted multiple dilated air-filled loops of small bowel, a decompressed colon, and rectal vault, with expected free air in the abdominal cavity after the minimally invasive procedure.

\footnotetext{
${ }^{1}$ College of Medicine, The Pennsylvania State University, Hershey, Pennsylvania, USA.

${ }^{2}$ Glickman Urological and Kidney Institute, Cleveland Clinic, Cleveland, Ohio, USA.

${ }^{3}$ Division of Minimally Invasive Surgery, Department of Surgery, College of Medicine, The Pennsylvania State University, Hershey, Pennsylvania, USA.

${ }^{4}$ Division of Urology, Department of Surgery, College of Medicine, The Pennsylvania State University, Hershey, Pennsylvania, USA.
} 
FIG. 1. (A) Cystogram demonstrating extravasation of contrast into the rectum and colon. (B) Noncontract CT scan revealing colovesicular fistula through the prostatectomy site with an $\sim 1.5 \mathrm{~cm}$ defect along the anterior aspect of the lower rectum at the level of the tip of the coccyx.
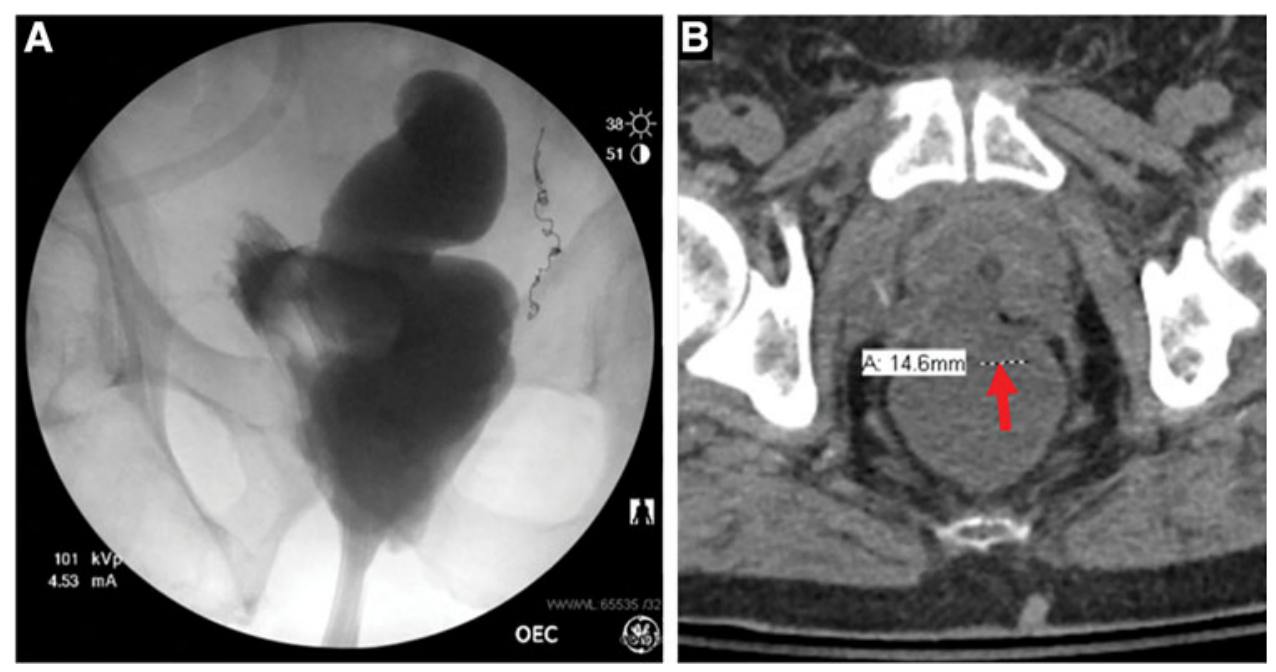

He acutely worsened after 6 days on bowel rest and developed gastrointestinal (GI) bleeding with hematochezia, gross hematuria, and an acute abdomen with hemodynamic instability. An emergent exploratory laparotomy revealed an unrecognized rectal injury, and abdominal washout with creation of a diverting transverse colostomy was performed. He remained critically ill in the ICU for several days requiring several reoperative procedures because of continued hemodynamic instability. These procedures included additional abdominal washouts and embolization of a bleeding accessory left pudendal artery resulting in eventual stability, diet tolerance, and return of bowel function.

On POD 16 after his exploratory laparotomy procedure, the patient was noted to have liquid draining per his rectum concerning for fistula. A CT scan and X-ray cystogram re- vealed an RVF and cystoscopy confirmed a large posterior fistulous connection (Fig. 1).

The patient was managed with a chronic indwelling catheter and was discharged to inpatient rehabilitation on hospital day 26 with monthly cystoscopic catheter exchanges confirming decreased fistula size. His prolonged recovery focused on optimizing nutritional repletion and physical reconditioning before definitive RVF repair.

The patient was evaluated by the minimally invasive general surgery service and given his extensive prior abdominal surgical history was considered a suitable candidate for attempted endoscopic RVF closure. A $9 \mathrm{~mm}$ colonoscope was inserted rectally and passed through the RVF (Fig. 2A). Three Hem-olok clips were removed from the fistulous tract and argon plasma coagulation was applied to the surrounding granulation
FIG. 2. (A) Fistula tract observed by the colonoscope with tip of the flexible cystoscope seen in the bladder and a Hem-o-lok clip seen in the 6 o'clock position.

(B) Suture line created through the OverStitch device along the fistula orifice. (C) Sutured fistula orifice suctioned into colonoscope with unfired Ovesco clip seen on the outside of the scope.

(D) Fistula orifice after placement of Ovesco clip.
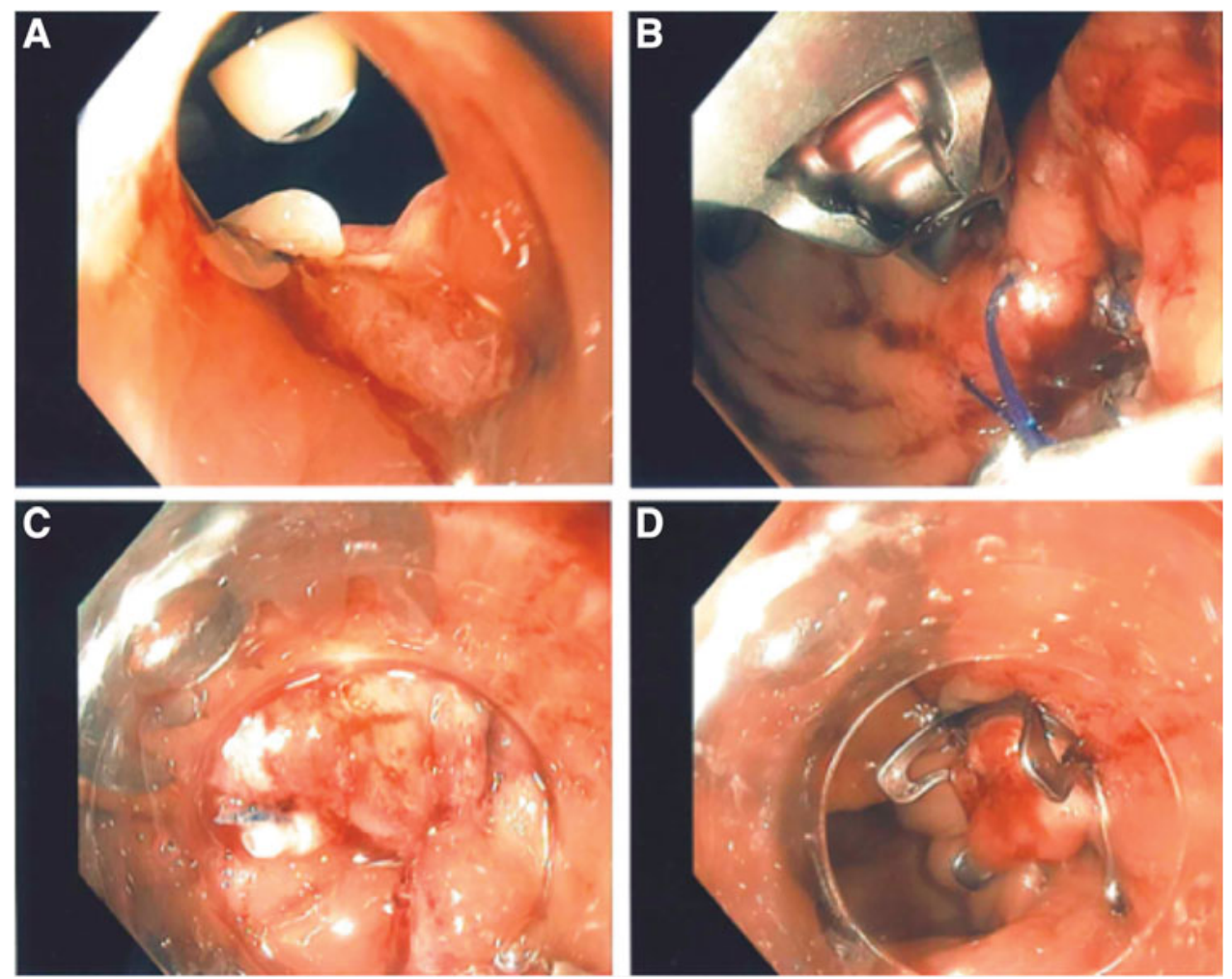
tissue. Urology provided simultaneous cystoscopic observation to avoid potential ureteral orifice involvement (Fig. 2A).

A novel technique called the Ovesco over OverStitch or The "Triple O" method was performed, which involved application of the OverStitch device along the fistula orifice followed by placement of an Ovesco clip overlying the OverStitch suture line (Fig. 2B-D). Intraoperative X-ray cystogram confirmed no contrast extravasation; however, follow-up barium enema study 1 month later revealed a persistent but improved RVF.

He would eventually need a total of four endoscopic procedures to achieve a complete and sustainable closure of his rectovesical fistula. The second and third procedures involved the removal of the existing Ovesco clip and placement of a new Ovesco clip. Intraoperatively no leak was identified after the placement of the new clips; however, recurrence of the fistula was found after each procedure at 1 and 3 months, respectively. During his fourth and final endoscopic procedure, the fistula tract was aggressively ablated before placement of another Ovesco clip and effective closure with no leaks was again achieved. Since the time of this procedure the patient has remained symptom free and has had a barium enema that showed no evidence of a persistent or recurrent rectovesical fistula (Fig. 3). He has subsequently had reversal of his colostomy with return of bowel continuity. He additionally remained catheter free during this endoscopic closure process and is surprisingly fully continent without need of an undergarment.

\section{Discussion and Literature Review}

RVF are uncommon entities with a variety of different etiologies, including inflammatory diseases, pelvic malignancy, or as consequence of iatrogenic injury or radiotherapy. ${ }^{1,2}$

Optimal surgical management for RVF is dependent on the underlying pathology report, site of the lesion, and preoperative status of the patient. Both open and laparoscopic procedures can be used for treatment with the goal of the operation being to resect and reanastomose the diseased bowel segment and close the bladder. ${ }^{1}$ However, as a result of the uncommon occurrence of RVF, there is limited information in this literature regarding the most effective surgical treatment. Colonic resection with primary anastomosis or diverting colostomy is currently the most widely used surgical treatment option for RVF. ${ }^{1}$ The York Mason posterior trans-sphincteric repair is another feasible and effective surgical approach that allows for excellent exposure of the fistulous tract and the possibility of avoiding fecal diversion. ${ }^{2}$

More recently, other less invasive endoscopic modalities have become potential alternatives of formal abdominal repair. The over-the-scope clip (OTSC) system is one such endoscopic approach with investigation occurring on the endoscopic closure of GI fistulae, including RVF. However, two factors have hindered researchers from comparing the various treatment modalities for closure of RVF, including the heterogeneity of the underlying etiologies causing RVF and the infrequency in which they occur. ${ }^{3}$

One meta-analysis by Weiland et al. found that using the OTSC system in the endoscopic closure of GI fistulae was safe, feasible, and had a clinical success rate of $69 \%$. In addition, repeat OTSC procedures after initial clinical failure may further increase the success rate. ${ }^{3}$ Another study by Mercky et al. found similar results in a retrospective study looking at 30 patients who had their digestive fistulae closed endoscopically with OTSC with an overall success rate of $71.4 \%$. Interestingly, $53 \%$ recovered with primary efficacy, but $20 \%$ required a subsequent endoscopic OTSC procedure to achieve clinical success. ${ }^{4}$ This is supported further by the case we present in this study with our patient needing a total of four procedures utilizing the OTSC system to achieve effective closure of his RVF.

Ultimately, RVF are rare complications of robot-assisted laparoscopic radical prostatectomies with no one corrective procedure standing out as superior in terms of efficacy. In an effort to provide patients with safe and effective procedures to treat RVF, it is important to use or at least discuss the option of endoscopic closure with OTSC as it has been found in multiple studies to be safe and effective with good clinical success rates.
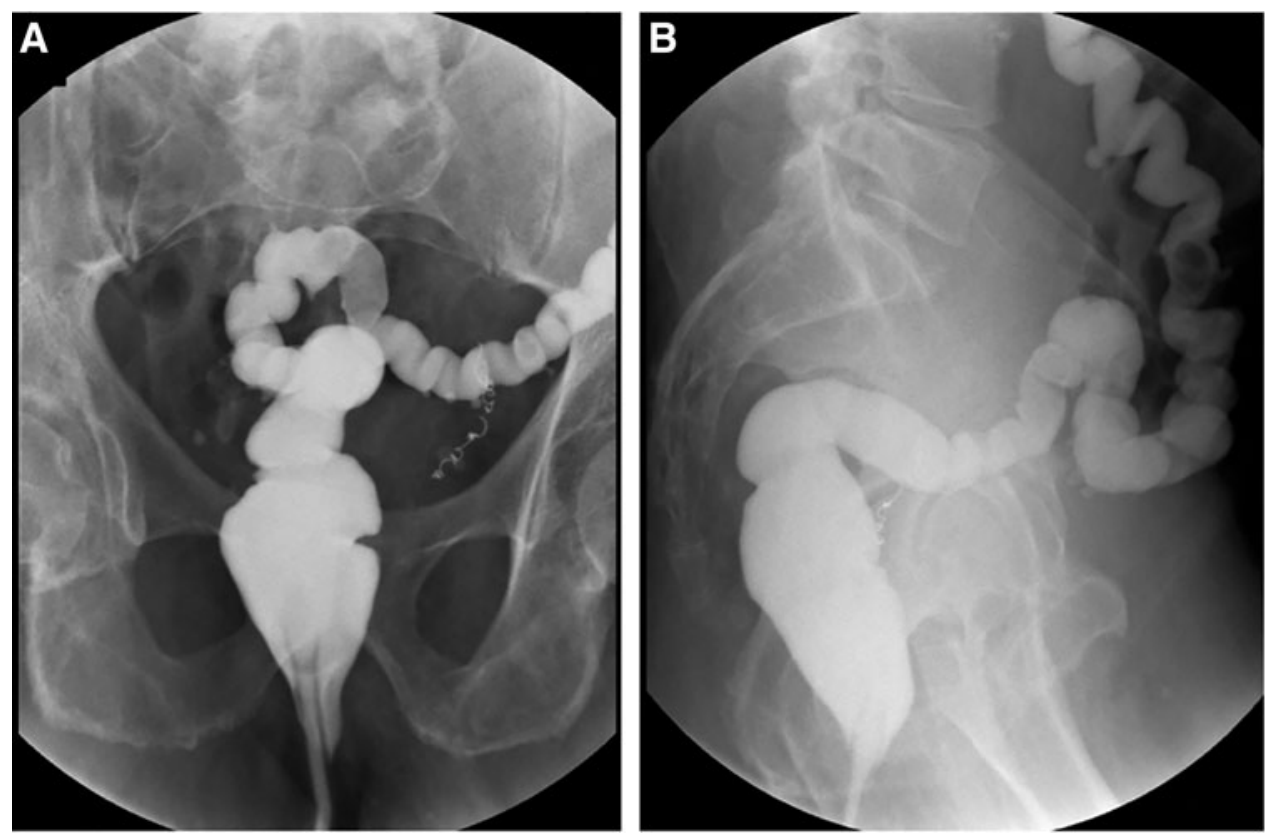

FIG. 3. X-ray watersoluble enema revealing no leak of contrast and no fistulous tract or filling of the bladder. (A) Coronal view. (B) Lateral view. 


\section{Conclusion}

We report a case of a rectovesical fistula in a 78-year-old man that was closed through a series of endoscopic procedures utilizing Ovesco clips on the OTSC system after the fistula developed after robot-assisted laparoscopic radical prostatectomy with a complicated postoperative course. RVF are traditionally treated surgically with removal of the fistula through bowel resection with or without temporary colostomy. These procedures are not benign and can have high rates of morbidities or mortality for patients without a guarantee of achieving closure. Therefore, endoscopic closure with Ovesco clips should be considered as a potential option for patients with a rectovesical fistula as it is a simple procedure with minimal risks that has minimal impact on a patient's well-being and still allows for subsequent open closure if needed.

\section{Disclosure Statement}

No competing financial interests exist.

\section{Funding Information}

This article was funded by the Ken and Bonnie Fund for Urologic Cancer Research.

\section{References}

1. Golabek T, Szymanska A, Szopinski T, et al. Enterovesical fistulae: Aetiology, imaging and management. Gastroenterol Res Pract 2013;2013:617967.

2. Falavolti C, Sergi F, Shehu E, et al. York Mason procedure to repair iatrogenic rectourinary fistula: Our experience. World J Surg 2013;37:2950-2955.
3. Weiland T, Fehlker M, Gottwald T, et al. Performance of the OTSC System in the endoscopic closure of gastrointestinal fistulae-A meta-analysis. Minim Invasive Ther Allied Technol 2012;21:249-258.

4. Mercky P, Gonzalez JM, Aimore Bonin E, et al. Usefulness of over-the-scope clipping system for closing digestive fistulas. Dig Endosc 2015;27:18-24.

Address correspondence to: Jay D. Raman, MD, FACS Division of Urology Department of Surgery College of Medicine The Pennsylvania State University 500 University Drive, MC H055 Hershey, PA 17033 USA

E-mail: jraman@pennstatehealth.psu.edu

$\begin{aligned} & \text { Abbreviations Used } \\ & \mathrm{CT}=\text { computed tomography } \\ & \mathrm{GI}=\text { gastrointestinal } \\ & \mathrm{OTSC}=\text { over-the-scope clip } \\ & \mathrm{RVF}=\text { rectovesical fistulae }\end{aligned}$

Cite this article as: Watts A, Kocher NJ, Pauli E, Raman JD (2020) Endoscopic closure of a large rectovesical fistula following robotic prostatectomy, Journal of Endourology Case Reports 6:3, 139-142, DOI: 10.1089/ cren.2019.0132. 